\title{
Erratum to: Exact Results for One Dimensional Fluids Through Functional Integration
}

\section{Riccardo Fantoni ${ }^{1}$}

Published online: 25 April 2016

(C) Springer Science+Business Media New York 2016

\section{Erratum to: J Stat Phys DOI 10.1007/s10955-016-1510-3}

In the article "Exact Results for One Dimensional Fluids Through Functional Integration" by Riccardo Fantoni the Eq. (57) of Sect. 8 should read

$$
C\left(x_{i}, x_{j}\right)=\frac{2}{\theta \sigma^{2}} w\left(x_{i}, x_{j}\right)=\prod_{k=i}^{j-1} A\left(\left|x_{k}-x_{k+1}\right|\right),
$$

with $x_{i} \leq x_{i+1} \leq x_{i+2} \leq \cdots \leq x_{j}$. So the interaction between particle $i$ and particle $j$ depends on how many particles lie between them. This is a particular non pairwise-additive interaction model. That is, taking $v=A \theta \sigma^{2} / 4$, the model fluid studied in Sect. 8 and the following subsections is one with a potential energy of the following form

$$
V_{N}=\sum_{i<j} \prod_{k=i}^{j-1} v\left(\left|x_{k}-x_{k+1}\right|\right)
$$

for a configuration with $x_{1} \leq x_{2} \leq x_{3} \leq \cdots \leq x_{N}$.

The online version of the original article can be found under doi:10.1007/s10955-016-1510-3.

$凶$ Riccardo Fantoni rfantoni@ts.infn.it

1 Dipartimento di Fisica, Università di Trieste, Strada Costiera 11, Grignano, 34151 Trieste, Italy 